\title{
Packing density of bi-disperse mixtures under one-dimensional compression
}

\author{
Giulia Guida ${ }^{*}$ and Francesca Casini $^{2}$ \\ ${ }^{1}$ Politecnico di Milano, Department of Civil and Environmental Engineering (DICA). Piazza Leonardo Da Vinci 32, 20133 Milan. \\ ${ }^{2}$ Università degli Studi di Tor Vergata, Department of Civil and Informatic Engineering (DICII). Via del Politecnico 1, 00133 Rome.
}

\begin{abstract}
This work aims to investigate the effect of fines in different type of bi-disperse grain size distributions in terms of minimum/maximum density, compressibility and crushing. The material adopted is the Light Expanded Clay Aggregate (LECA), an artificial granular material characterized by light, porous and crushable grains. The bi-disperse grading are firstly analysed in terms of packing density, measuring experimentally the minimum and the maximum porosity for different combination of sizes and volume proportions. Then, some selected mixtures are subjected to one-dimensional compression tests up to high pressures. Evolution of grain size due to grain crushing phenomena and compressibility are therefore measured and interpreted. Finally a theoretical model is adopted in order to predict the mechanical material response accounting grain crushing and granular microstructure.
\end{abstract}

\section{Introduction}

The state of density of a granular material rules its mechanical, thermal and hydraulic behaviour. The density state can be expressed as inversely proportional to the porosity index $n$, defined as the volume of voids over the total reference volume. The minimum $n_{\min }$ and maximum $n_{\max }$ porosity limits of a granular material are intrinsic properties and can be practically evaluated by standard procedures $[1,2]$. The greater is the range of grain sizes, the greater will be the density and the lower the minimum and maximum porosity $[3,4]$. Analogously, the more irregular is the shape of the grains the lower will be the density of the material, because irregularities hinders particle mobility and rearrangements $[5,6]$. From a theoretical point of view [7], the variation of the state of density is mainly due to irreversible phenomena such as: rearrangement/dilation inducing a decrease/increase of the material porosity [8]; grain crushing [9] that, increasing the polydispersity of the grain size distribution, may lead to a decrease of the porosity maximum and minimum intrinsic limits. In this work, the density state of bi-disperse mixtures are investigated both experimentally and theoretically. The effects of the percentage of fines and of the ratio between the coarse and the fine particles are explored, as well as the behaviour of some selected mixtures subjected to one-dimensional compression and grain crushing.

\section{Packing density}

Bi-disperse distributions are composed by grains of two distinct sizes, coarse namely $D$ and fine $d$, where $D>d$. The quantitative proportion of the two volume fraction is

*e-mail: giulia.guida@polimi.it

A video is available at https://doi.org/10.48448/8c1q-kf15 expressed by the fine percentage in volume $V=V_{d} / V_{t o t}$, where $V_{\text {tot }}=V_{d}+V_{D}$ and $V_{d}, V_{D}$ are the percentage by volume of fractions $d$ and $D$ respectively. The choice to express the fraction proportion by volume is due to the material adopted, the light expanded clay aggregate (LECA), which has a unit weight that increases with decreasing particle diameter [10] due to the presence of intra-porosity [11]. Figure 1 shows experimental measurements of maximum and minimum void ratios for different type of bidisperse grading, characterised by a ratio between diameters $R=D / d$ and a percentage of fines $V$. The experimental evidences coming from Fig. 1 highlight how, especially for grater $R$, the material reaches a greater packing density, both for the maximum and minimum porosity measurements, in correspondence of $V=25-50 \%$. This is in accordance to several theoretical and experimental studies $[12,13]$. Note that in the case of monodisperse distribution, when $V=0$ or $V=1$, that means a mixture composed only by coarse or fine grains respectively, the porosity limits assume different values based on the size, higher for fines grains. This is due to a different grain shape between the fine and the coarse fractions. Figure 2 shows the outline morphology of a set of grains selected randomly from the different fractions of untested LECA material, to not have dependency on loading stages. Increasing the size fraction, grains present smoother, more rounded and circular outline, that enable a better rearrangement. An increasing irregularity of grain morphology leads indeed to less packing density and to greater values of maximum and minimum porosity [14-16].

\section{One-dimensional compression}

Two bi-disperse mixtures among those reported in Fig. 1 are selected to be tested under one-dimensional compres- 

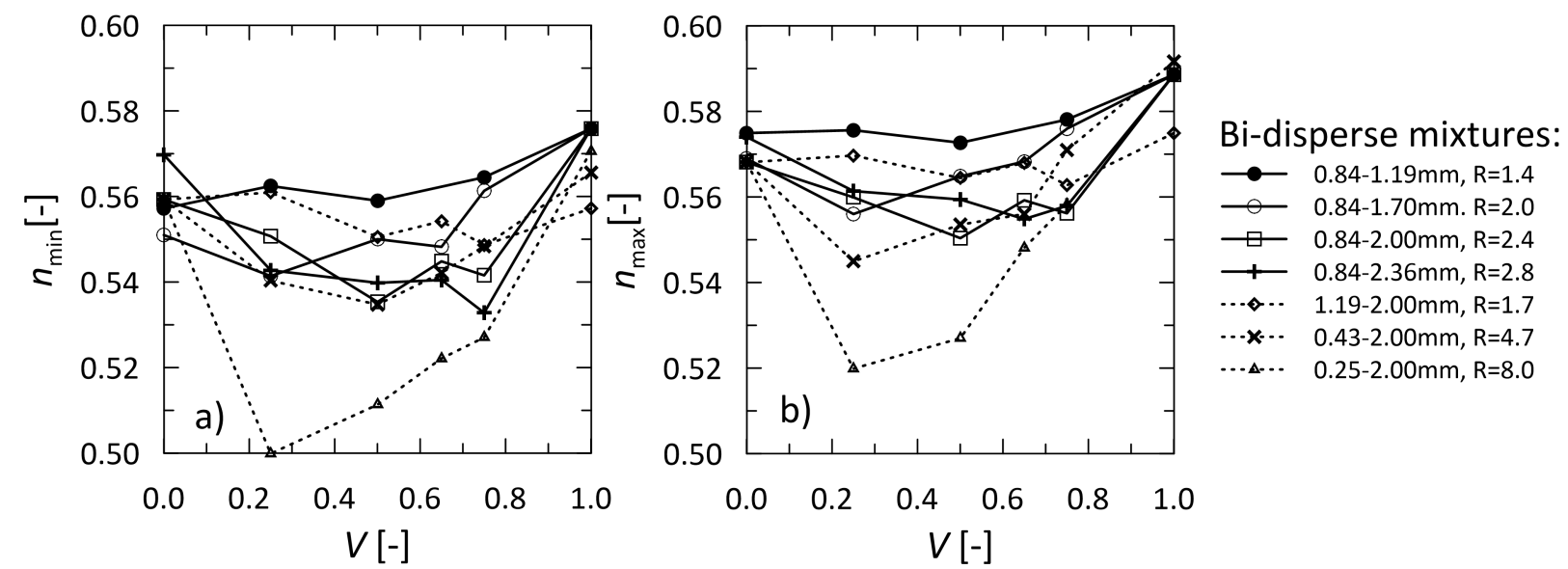

Figure 1. Measures of (a) minimum and (b) maximum void ratios for bi-disperse mixtures as a function of $V$, for different values of $R$.

sion: (1) with $D=2.00 \mathrm{~mm}, d=0.43 \mathrm{~mm}, R=4.7$ and $V=0.4$ (Fig. 2a); (2) with $D=2.00 \mathrm{~mm}, d=0.25 \mathrm{~mm}$, $R=8$ and $V=0.4$ (Fig. 2d). Those mixtures are dry pluviated into the oedometer with a diameter of $2.5 \mathrm{~cm}$ and an height of $5 \mathrm{~cm}$, and subjected to a maximum vertical pressure of $54 \mathrm{MPa}$. The same procedure is adopted on the two monodisperse samples, $\mathrm{MONO}_{D}$ and $\mathrm{MONO}_{d}$, characterised by the coarse and fine fractions respectively of the bi-disperse mixture (Fig. 3b,e). Figures 3a,d and b,e report the cumulative grain size distributions (CGSD) obtained by sieving of the bi-disperse and monodisperse mixtures respectively, that at different loading stages evolve due to grain crushing. Note that the bi-disperse CGSD, at high pressure, evolves similarly to $\mathrm{MONO}_{D}$ for the coarse fractions, and similarly to $\mathrm{MONO}_{d}$ for the fine fractions. Monodisperse CGSDs start crushing at lower pressures compared to bi-disperse, with the tendency to assume a fractal grain size distribution increasing the vertical stress [17]. Figures $3 c$,f show the evolution of porosity $n$ as a

\section{Fractions $[\mathrm{mm}]$}

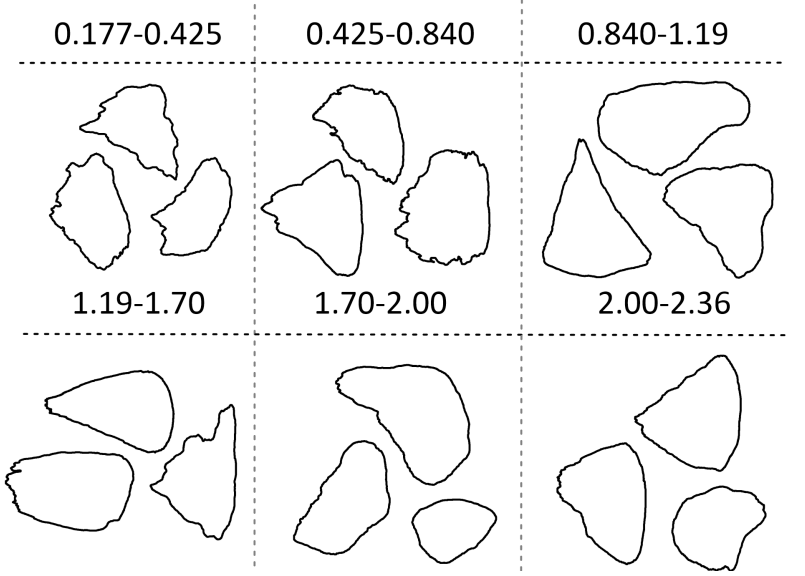

Figure 2. Quantitative comparison of LECA grain shape outline for different size fractions. function of vertical stress $\sigma_{v}$. Bi-disperse mixtures, both (1) and (2), in accordance to what observed in Fig. 1, assume a lower initial porosity than monodisperse samples due to an higher polydispersity that allows the grains to rearrange more efficiently. The greater packing density of the bi-disperse mixtures increases the grains confinement and inhibits crushing. Indeed, bi-disperse samples yield for greater vertical stresses and explicate less compressibility than monodisperse samples.

The model developed by [7] is here applied in order to predict the compressibility response of the mixture. In particular the model links the variation of porosity $\dot{n}$ to the variation of the maximum and the minimum limits of porosity due to grain crushing, and to the variation of the index $\tau_{n}$ (equal to 1 when $n=n_{\max }$ and to 0 for $n=n_{\text {min }}$ ), [18]) due to irreversible rearrangement:

$$
\dot{n}=\tau_{n} \dot{n}_{\max }+\left(1-\tau_{n}\right) \dot{n}_{\min }+\dot{\tau}_{n}\left(n_{\max }-n_{\min }\right) .
$$

For a sake of simplicity, in this work the index $\tau_{n}$ has assumed to be constant and equal to 1 for the whole test. This means that the variation of porosity can only be due to phenomena related to grain crushing. The intrinsic values of $n_{\max / \min }$ are defined as a function of grainsize polydispersity, quantified by the index of grading $I_{G r}$ :

$$
n_{\max / \min }=n_{\max / \min }^{M O N O} I_{G r}^{-\alpha},
$$

where $n_{\max / \min }^{M O N O}$ is the minimum and maximum porosity at zero stress of a mono-disperse grading and $\alpha$ is a dimensionless model parameter. The index of grading $I_{G r}$ is defined by [7] as the geometrical standard deviation of the particle size distribution, it is dimensionless and describes the spread of grain size distribution about its geometrical mean. Figure 4 shows the evolution of the index of grading $I_{G r}$ with stress for the sample tested. All the monodisperse samples begin from an unitary value at zero stress and then grow up as fast as crushing phenomena occurred. A comparison between experimental data and model prediction is reported in Fig. 3c,f in terms of evolution of porosity as a function of vertical stress. The model aims reasonably well to catch the compressibility features, especially in the 
(a)

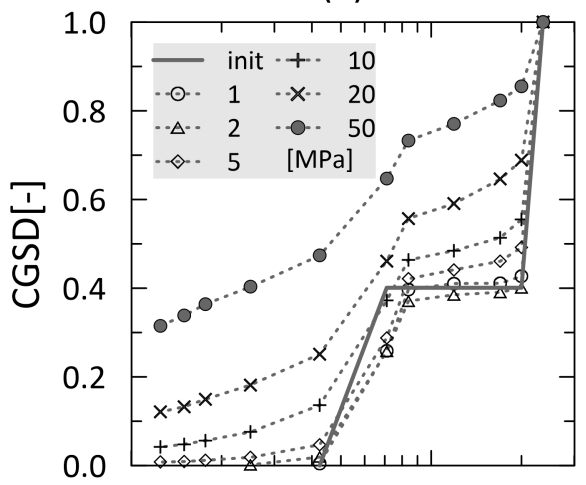

0.1 (d)

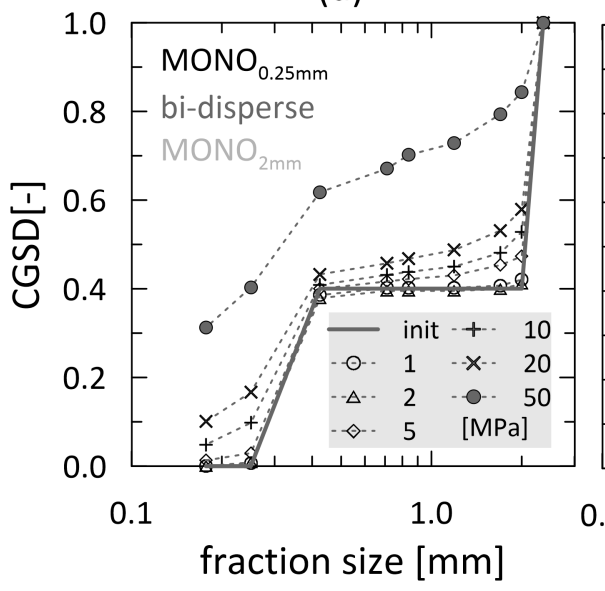

(b)

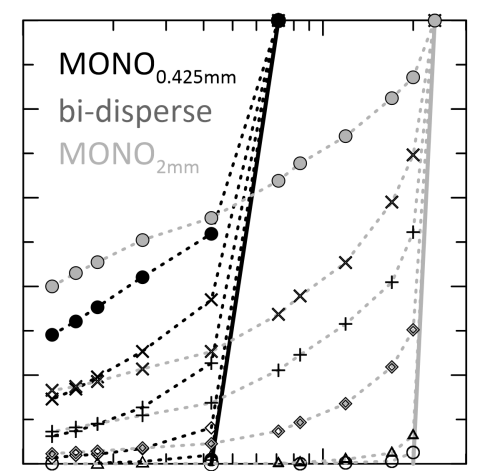

0.1

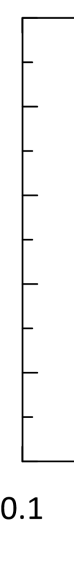

(e)

1.0

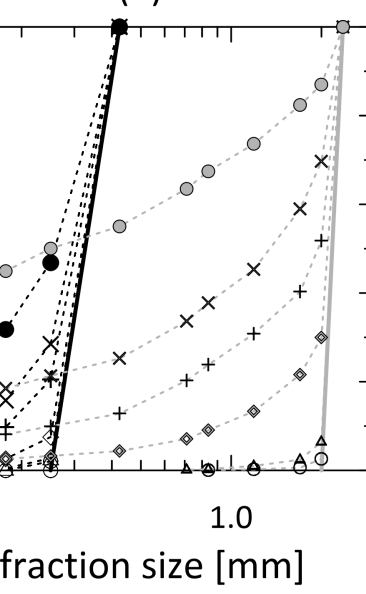

(c)
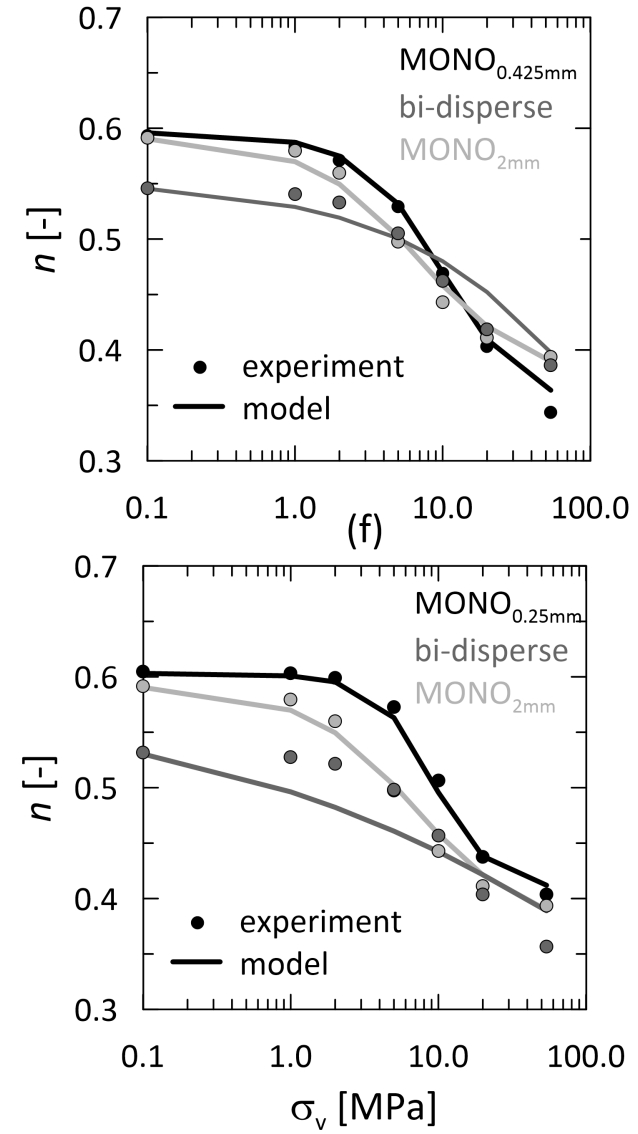

Figure 3. (a-b,d-e) CGSD evolution under 1D compression for the bi-disperse grading mixture 1(a) and 2(d), compared to the grading evolution of their relative mono-disperse ones: (b) $\mathrm{MONO}_{0.425 m m}, \mathrm{MONO}_{2.0 \mathrm{~mm}}$ and (e) $\mathrm{MONO}_{0.25 m m}, \mathrm{MONO}_{2.0 \mathrm{~mm}}$. (c,f) Experimental and model prediction compressibility curves.

monodisperse cases, in which porosity variation are effectively strongly linked to the phenomenon of grain crushing. Table 1 reports the model parameters adopted. The model predictions reported in Fig. 3c,f are not smooth be-

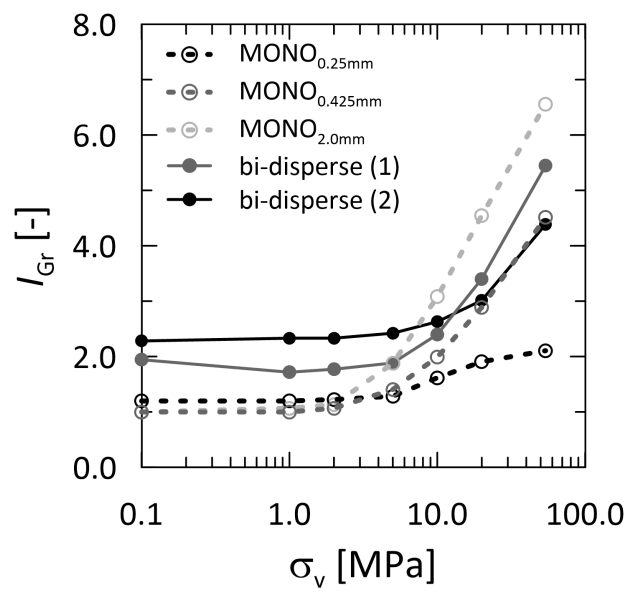

Figure 4. Evolution of the grain size distribution polydispersity, by the index of grading, with vertical stress along the onedimensional compression. cause they are related to the polydispersity of the grain size distribution $\left(I_{G r}\right)$ which was measured just for fixed values of load applied. Since $\tau_{n}=1$, the variation of $n$ depends only by $n_{\max }^{M O N O}$, that is defined in accordance to the measurements reported in Fig. 1. The model parameter $\alpha$ was calibrated by trial, and for the bi-disperse mixtures it assumes values in between those of the monodisperse of the coarse and fine constitutive fractions.

Table 1. Model parameters.

\begin{tabular}{llll} 
& $n_{\max }^{\text {MONO }}$ & $\alpha$ & $\tau_{n}$ \\
\hline $\mathrm{MONO}_{0.25 m}$ & 0.67 & 0.65 & 1 \\
$\mathrm{MONO}_{0.43 m m}$ & 0.59 & 0.33 & 1 \\
$\mathrm{MONO}_{2.0 \mathrm{~mm}}$ & 0.57 & 0.20 & 1 \\
bi-disperse $_{0.43-2.0 \mathrm{~mm}}$ & 0.59 & 0.22 & 1 \\
bi-disperse $_{0.25-2.0 \mathrm{~mm}}$ & 0.67 & 0.4 & 1 \\
\hline
\end{tabular}

\section{Conclusions}

This paper reports the main results of an experimental and theoretical investigation on bi-disperse mixtures of LECA material. As expected bi-disperse mixtures explicate a greater packing density compared mono-disperse grading, 
with a tendency to reach a maximum value for a percentage of fine ranging between $25-50 \%$. However, not only the grain size distribution affected the packing density, also particles morphology: the more irregular outline of the fine fractions determined less dense configurations. Two bi-disperse mixture with a $40 \%$ of fines are then selected to be tested under one-dimensional compression up to a vertical pressure of $54 \mathrm{MPa}$. The comparisons between the results of the bi-disperse mixtures and those of two monodisperse grading composed by only the fine and the coarse fraction respectively let emerge some interesting features: (i) the evolution of the cumulative grain size distribution due to crushing follows, especially at high pressures, a behaviour in between the two mono-disperses; (ii) the bidisperse mixture leads to an initial denser configuration, to a greater resistance to grain crushing phenomena and to a less compressibility response. This behaviour tendency seems to be as more enhance increasing the ratio $R$ between coarse and fine fractions. Finally, the experimental evidences gathered are used to validate a compressibility model for granular material with crushable grains recently developed.

\section{References}

[1] ASTM, Test Methods for Maximum Index Density and Unit Weight of Soils Using a Vibratory Table. In Annual Book of ASTM Standards, West Conshohocken, PA. (1996)

[2] ASTM, Test Methods for Minimum Index Density and Unit Weight of Soils and Calculation of Relative Density. In Annual Book of ASTM Standards, West Conshohocken, PA. (1996)
[3] D.M. Burmister, The importance and practical use of relative density in soil mechanics, In Proc. American society for testing materials (1948)

[4] N. Peronius, T. Sweeting, Pow. Tech. 42, 2 (1985)

[5] G.C. Cho, J. Dodds, J.C. Santamarina, J. Geotech. Geoenv. Eng. 132, 5 (2006)

[6] G. Guida, D. Sebastiani, F. Casini, S. Miliziano, Géot. Lett. 9, 4 (2019)

[7] G. Guida, I. Einav, B. Marks, F. Casini, Int. J. Sol. Str. 187 SI: Mech. Granular Materials (2020)

[8] A. Tengattini, A. Das, I. Einav, Géotecnique 66, 9 (2016)

[9] Itai Einav, J. Mech. and Phys. Sol. 55, 6 (2007)

[10] F. Casini, G.M.B. Viggiani, Experimental investigation of the evolution of grading of an artificial material with crushable grains under different loading conditions. In Proc. of the 5th international symposium on deformation characteristics of geomaterials, Seoul, Korea (2011)

[11] G. Guida, F. Casini, G.M.B. Viggiani, E. Andò, G. Viggiani, Géot. Lett. 8, 2 (2018)

[12] P.V. Lade, C.D. Liggio Jr., J.A. Yamamuro, Geot. Test. J. 21, 4 (1998)

[13] T. Shire, C. O’Sullivan, K.J. Hanley, Gran. Matt. 18, 3 (2016)

[14] M. Cubrinovski, K. Ishihara, Soil Found. 42, 6 (2002)

[15] R.S. Farr, R.D. Groot, J. Chem. Phys. 131, 24 (2009)

[16] D.M. Wood, K. Maeda. Acta Geotecnica 3, 1 (2008)

[17] X. Zhang, B.A. Baudet, The multi-fractal nature of soil particle size distribution, In Geomechanics from micro to macro, Taylor \& Francis Group, London (2015)

[18] M. Rubin, I. Einav, Int. J. Eng. Sc. 49, 10 (2011) 